\title{
Generating Vector Fields of the Metric Semi-Symmetric Connection on Almost Hyperbolic Kaehlerian Manifolds
}

\author{
Umraw Singh Negi ${ }^{1 *} \cdot$ Preeti Chauhan ${ }^{1}$ \\ ${ }^{1}$ Department of Mathematics, H.N.B. Garhwal University (A Central University), S.R.T. Campus Badshahi Thaul, \\ Tehri Garhwal - 249 199, Uttarakhand, India
}

Corresponding Author Email: $\underline{\text { usnegi7 @gmail.com }}$

Received: 17.4.2021; Revised: 11.5.2021; Accepted: 14.5.2021

CSociety for Himalayan Action Research and Development

\begin{abstract}
Negi and Semwal (2011), have studied on almost Kaehlerian conformal recurrent and symmetric Manifolds. In this paper, we have defined and calculated Generating vector fields of the metric semi-symmetric connection (MS-Sc) on almost Hyperbolic Kaehlerian Manifolds and its some theorems established.
\end{abstract}

Keywords: Geodesic Line • Induced and Isotropous Vector Fields • Hyperbolic Kaehlerian Manifolds

Mathematics subject classification 2010: 53A30, 32C15, 53C55.

\section{Introduction:}

A $\quad \boldsymbol{n}(=\mathbf{2 m})$ dimensional Riemannian space

$M$, with metric tensor (

The geodesic line of $\mathbf{L}-\mathbf{C c}$ is differentiated by the following [Mizusawa and Koto (1960)]:

$$
v^{k} \dot{\nabla}_{k} v^{j}=0
$$

where $v^{k}$ locates a module of tangent vector field of the geodesic line.

The modules of MS-Sc are known as:

$$
\Gamma_{i k}^{a}=\left\{\begin{array}{c}
a \\
i k
\end{array}\right\}+p_{i} \delta_{k}^{a}-p^{a} g_{i k}
$$

where $p_{i}$ and $p^{a}$ are known as producer or induced vector field of MS-Sc. The torsion tensor is equal to [Goldberg (1956)]:

$$
\mathrm{T}_{i k}^{a}=p_{i} \delta_{k}^{a}-p^{k} \delta_{i}^{a}
$$

if (1.3) holds for $v^{k}$, then:

$$
p_{k} v^{k} v^{j}=p^{j} v_{k} v^{k}
$$

while, we reflect on a Riemannian space and its metrics is absolutely exact then (1.3) reduces:

$$
p_{k}={ }^{\alpha} p_{k}
$$

where ${ }^{\alpha}$ is a scalar function. 
But here effect of the function $\quad \alpha$ is the comparatively concurrent involving the geodesic line and induce vector field of MS-Sc. Allocate us indicate the machinist of covariant differentiation in the direction by

$$
\nabla \text {, then }
$$

$$
\begin{aligned}
& \nabla_{k} v_{j}=\dot{\nabla}_{k} v_{j}-\alpha\left(v_{k} v_{j}-g_{k j}\right) v \\
& \dot{\nabla}_{k} p_{j}=\alpha_{k} v_{j}+\alpha \dot{\nabla}_{k} v_{j},
\end{aligned}
$$

where $v$ sets used for scalar four-sided figure of the vector $v_{k}$ with $\alpha_{k}=\partial \alpha / \partial x^{k}$.

Now modules of Riemannian curvature tensor of MS-Sc can be communicated herein mode:

$$
R_{i j k l}=K_{i j k l}+g_{i k} p_{l j}-g_{i l} p_{k j}+g_{j l} p_{k i}-g_{j k} p_{l i}
$$

where $K_{i j k l}$ signify Riemann curvature tensor module of $\mathbf{L}-\mathbf{C c}$ and contraction $p_{k j}$ locates for:

$$
p_{k j}=\dot{\nabla}_{k} p_{j}-p_{j} p_{k}+\frac{1}{2} \mathrm{p}_{s} p^{s} g_{j k}
$$

The tensor $\quad p_{k j}$ is symmetric if and only if

\section{$p_{j}$ is a gradient, that means}

$$
\alpha_{k} v_{j}-\alpha_{j} v_{k}=\alpha\left(\dot{\nabla}_{j} v_{k}-\dot{\nabla}_{k} v_{j}\right)
$$

Or, equivalently

$$
\frac{\partial v}{\partial x^{k}}=\frac{2}{a}\left(\mu v_{k}-v \alpha_{k}\right)
$$

where ${ }^{\mu}$ stands for $\alpha_{k} v^{k}$.

Since the appearance (1.5), we simply obtain [Yano (1965)]:

$$
\begin{aligned}
& R_{j k}=K_{j k}+(2-n) p_{k j}-g_{j k} p_{s,}^{s} \\
& g_{k j} p_{s}^{s}=K_{j k}-R_{j k}-(n-2) p_{j k}, \\
& n p_{s}^{s}=K-R-(n-2) p_{s}^{s}, \\
& p_{s}^{s}=(K-R) / 2(n-1), \\
& p_{k j}=\frac{K_{j k}-R_{j k}}{n-2}-\frac{K-R}{2(n-1)(n-2)} g_{j k} .
\end{aligned}
$$

Here $K_{j k}, K$ and $R_{j k}, R$, indicate Ricci tensor, curvature scalar for the Levi-Civita connection respectively.

Here, comprise the curvature tensor equation (1.5) convince the entire statistical conditions which are mainly universal for Riemannian curvature tensors to exist skew-symmetric into primary both indices regular in modify sets of primary with next couple of indices and to convince of initial Bianchi identity. The entire properties be fulfilled iff the induced vector field is an inclined [Prvanovic and Pusic (1995)].

There holds:

$$
v^{i} \dot{\nabla}_{i} v_{k}=0 \text { and } p^{i} \dot{\nabla}_{i} p_{k}=p^{i} \dot{\nabla}_{k} p_{i}=\varphi p_{k}=\varphi \alpha v_{k}
$$

Next, concern the Ricci character for connection to the producer and then we find:

$$
v \alpha_{j}-\varphi v_{j}=0 .
$$

Then there yields, in view of (1.7), $\frac{\partial v}{\partial x^{k}}=0$, and from (1.8), we have

$$
\alpha_{k}=\frac{\varphi}{v} v_{k}, \quad \text { or } \quad \alpha_{k}=f p_{k} .
$$

This represents every three vectors are mutually comparative. Therefore: 


$$
p_{s} p^{s}=\alpha^{2} v
$$

Besides:

$$
\begin{aligned}
\frac{\partial\left(p_{s} p^{s}\right)}{\partial x^{k}} & =p^{s} \dot{\nabla}_{k} p_{s}+p_{s} \dot{\nabla}_{k} p^{s}=p^{s} \alpha_{k} p_{s}+\alpha p^{s} \dot{\nabla}_{k} v_{s} \\
& =2 \alpha_{k} p_{s} p^{s}+2 \alpha p^{s} \dot{\nabla}_{k} v_{s}=2 \alpha_{k} p_{s} p^{s}+2 \alpha^{2} v^{s} \dot{\nabla}_{k} v_{s} \\
& =2 p_{s} p^{s} \alpha_{k}=2 \alpha^{2} \alpha_{k} v .
\end{aligned}
$$

As $\dot{\nabla}_{k} v^{s} v_{s}=0$ and consequently $v^{s} \dot{\nabla}_{k} v_{s}=0$,

Then, on the other side

$$
\begin{aligned}
\frac{\partial\left(p_{s} p^{s}\right)}{\partial x^{k}} & =2 p^{s} \dot{\nabla}_{k} p_{s}=2 \alpha_{k} p^{s} p_{s}=2 \alpha^{2} v \alpha_{k} \\
& =2 \alpha v \alpha_{k^{*}}
\end{aligned}
$$

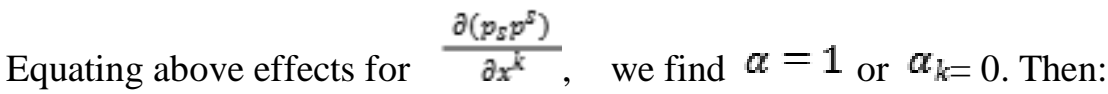

(1) $\left(p_{k}\right)$ and $\left(v_{k}\right)$ are equivalent, both slopes, both of regular span.

or

(2) $\left(p_{k}\right)$ and $\left(v_{k}\right)$ are smooth vectors, equally of regular span and equally slopes.

Definition (1.1): In a Riemannian manifold, the curvature tensor of MS-Sc fulfilled entire extremely regular statistical conditions of the L-Cc and tangent vector fields of the geodesic lines are smooth inclines of constant length.

\section{Generating Vector Fields of the Metric Semi-Symmetric Connection on Almost Hyperbolic Kaehlerian Manifolds:}

An almost hyperbolic Kaehlerian manifolds are 2n-dimensional pseudo-Riemannian manifolds, capable by a non-disintegrate formation tensor $\quad F_{j}^{i}$ gratifying:

$$
F_{j}^{i} F_{k}^{j}=\delta_{k}^{i}, \quad F_{i j}=-F_{j i}, \quad \dot{\nabla} F_{i j}=0 .
$$

Here, we know that a almost hyperbolic Kaehlerian manifolds is in fact a product manifold, but its covariant structure tensor is skew-symmetric. Besides, the structure tensor has $\mathrm{n}$ linearly independent Eigen vectors, by the fact of skew-symmetry of the structure, it sends any vector into an orthogonal vector and Eigen vectors of the structure are consequently, self-orthogonal. In any point of a hyperbolic almost Kaehlerian manifold, its tangent space can be spanned by these self-orthogonal vectors, it is its adapted basis. It is obvious that these are two Eigen subspaces of equal dimension, for two structure's eigenvalues, 1 and -1. on both these invariant subspace, the metric tensor vanishes. Actually, a hyperbolic almost Kaehlerian manifolds in any point is a product of two totally geodesic subspaces. This is the reason to investigate geodesic lines of this kind of space. Here we may have such geodesic lines which are minimizing the distance between two different points up to zero, from one point, we may reach another point instantly along such a geodesic line.

Theorem (2.1): Proved that Riemannian spaces curvature scalar of Levi-Civita connection of MS-Sc and F-connection are commonly equivalent.

Proof: We have MS-Sc of an almost hyperbolic Kaehlerian manifolds contain the torsion tensor:

$$
T_{i j}^{k}=p_{i} \delta_{j}^{k}-p_{j} \delta_{i}^{k}+q_{i} K_{j}^{k}-q_{j} F_{i}^{k},
$$

where $p_{i}$ and $q_{i}$ are modules of positive vector fields. If this connection to be a metric one, then its modules: 


$$
H_{i k}^{a}=\left\{\begin{array}{c}
a \\
i \quad k
\end{array}\right\}+p_{i} \delta_{k}^{a}-p^{a} g_{i k}-q_{k} F_{i}^{a}
$$

If this signify that $\nabla F=0$. then

$$
q_{j}=-\frac{n}{2} p_{a} F_{j}^{a}, \quad p_{a} F_{j}^{a}=-\frac{2}{n} q_{j}
$$

Then we can denote:

$$
H_{i k}^{a}=\Gamma_{i k}^{a}-q_{k} F_{i}^{a}
$$

where $\Gamma_{i k}^{a}$ exists module of Riemannian spaces and module of identical on the adjunct pseudoRiemannian manifolds, fulfilling forms of definition (1.1) [Gray (1967)].

At present, compute the coefficients of (2.3), we get:

$$
\begin{aligned}
\bar{R}_{i j k l}= & R_{i j k l}-F_{j i}\left(\dot{\nabla}_{l} q_{k}-\dot{\nabla}_{k} q_{l}\right)+ \\
& +q_{k}\left(p_{j} F_{l i}+\frac{2}{n} q_{j} g_{l i}+\frac{2}{n} q_{i} g_{l j}+p_{i} F_{j l}\right)- \\
& -q_{l}\left(p_{j} F_{k i}+\frac{2}{n} q_{j} g_{k i}+\frac{2}{n} q_{i} g_{k j}\right)+p_{i} F_{j k}
\end{aligned}
$$

By $R_{i j k l}$ indicate a module of curvature tensor gratifying the def (1.1) and $\bar{R}_{i j k l}$ is skew-symmetric into initial indices. $\bar{R}_{i j k l}$ is consistent in growing places of primary and next couple of indices iff the tensor ( $\left.p_{l} q_{k}+q_{l} p_{k}\right)$ is skew-symmetric. Then:

$$
p_{k} p^{k} q_{l}=-p^{k} q_{k} p_{l}
$$

As the vectors $p^{k}$ and $q^{k}$ are mutually orthogonal, there yields $p_{k} p^{k}=0$. Its indicates the producer on the Riemannian spaces of F-connection is an isotropous slope and harmony through declaration of definition (1.1). Therefore vector $q_{k}$ as well isotropous slope.

Theorem (2.2): The curvature tensor of a MS-Sc and F-connection on the almost hyperbolic Kaehlerian manifolds invariable in varying sets of primary and next couple of index and making the primary Bianchi identity iff the producers on association are isotropous i.e. $\dot{\nabla}_{\alpha} p^{a}=0$ and conversely.

Proof: We have from (2.3), the pseudo-Riemannian manifolds structures and $v^{i}$ is satisfied following:

$$
\begin{aligned}
& p_{j} \delta_{k}^{i} v^{j} v^{k}-p^{i} q_{j} v^{j} v^{k}-q_{k} F_{j}^{i} v^{j} v^{k}=\frac{2}{n} p_{j} v^{j} v^{i} \\
& \left(p_{j} v^{j}-v_{j} v^{j}-\frac{2}{n} p_{j} v^{j}\right) v^{i}=-q_{k} v^{k} u^{i}, \\
& u^{i}=-F^{j i} v_{j}=F^{i j} v_{j .}
\end{aligned}
$$

where

Then, from (2.7),

$$
u^{i}=\alpha v^{i}, \quad \text { or } \quad q_{k} v^{k}=0 .
$$

If the eigen values 1 or $\mathbf{- 1}$, therefore $q_{k} v^{k}$ are equals to:

$$
\frac{n-2}{n} p_{j} v^{j}-v_{j} v^{j}=\frac{n-2}{n} p_{j} v^{j} \text {. }
$$

and from (2.8), correspond the vectors modified:

$$
p=p^{a} l_{a}+p^{\hat{b}} l_{\hat{b}},
$$

where $l_{\hat{h}}$ are eigen vectors, used for eigen values $\mathbf{- 1}$, we get:

$$
q=-\frac{2}{n} p^{a} l_{a}+\frac{2}{n} p^{\hat{b}} l_{\hat{b}} \quad \text { and } \quad v=v^{a} l_{a}+v^{\hat{b}} l_{\hat{b}} .
$$

Then (2.8) gives: 


$$
q_{k} v^{k}=\frac{2}{n}\left(p^{\hat{b}} v^{a}-p^{a} v^{\hat{b}}\right) g_{a \hat{b}}=0,
$$

That is fulfilled; as well as $v$ is comparative to $p$ and isotropous.

While the almost hyperbolic Kaehlerian manifolds induced vector fields of similar line containing several its autoparallel lines in ordinary with $\mathbf{L}$-Cc is isotropous, (1.6) appears that procedure:

$$
p_{k j}=\dot{\nabla}_{k} p_{j}-p_{k} p_{j}
$$

and

$$
\dot{\nabla}_{s} p^{s}=p_{s}^{s}=\frac{K-R}{2(n-1)}
$$

Constricting the tensor $\left(\dot{\nabla}_{k} q_{l}-\dot{\nabla}_{l} q_{k}\right)$ through the tensor $F_{b}^{l}$, we attain

$$
\begin{aligned}
& -\frac{2}{n} \dot{\nabla}_{k} p_{b}+\frac{2}{n} F_{b}^{l} F_{k}^{a} \dot{\nabla}_{l} p_{a}=-\frac{n}{2} \dot{\nabla}_{k} p_{b}+F_{b}^{l} \dot{\nabla}_{l} q_{k} \\
& \frac{n-4}{2 n} \dot{\nabla}_{k} p_{b}=F_{b}^{l}\left(\dot{\nabla}_{l} q_{k}-\frac{2}{n} F_{k}^{a} \dot{\nabla}_{l} p_{a}\right) .
\end{aligned}
$$

and

Contracting the last relation with $g^{k b}$, we obtain:

$$
\frac{n-4}{2 n} \dot{\nabla}_{a} p^{a}=-\frac{2}{n} \dot{\nabla}_{a} p^{a}+\frac{2}{n} p_{a} p^{a}=0 \text {. }
$$

If $n>4$, then

$$
\dot{\nabla}_{a} p^{a}=0 \text {. }
$$

Then, by (2.12), $\quad K=R$.

On the other hand, from (2.5) and the shape of the curvature tensor, we can obtain that:

$$
\begin{aligned}
& \bar{R}=R+F^{l k}\left(\dot{\nabla}_{l} q_{k}-\dot{\nabla}_{k} q_{l}\right) \\
& \bar{R}=R+\frac{2}{n}\left(\dot{\nabla}_{l} p^{l}+\dot{\nabla}_{l} p^{l}\right)=R+\frac{4}{n} \dot{\nabla}_{l} p^{l} .
\end{aligned}
$$

Therefore this tensor to gratify initial Bianchi identity with appearance for warp tensor, after that established:

$$
\dot{\nabla}_{a} p^{a}=0 .
$$

Hence theorem (2.2) is proved.

\section{References:}

Goldberg SI (1956). Note on projectively Euclidean Hermitian spaces, Proc. Nat. Acad. Sci. U.S.A., pp. 128-130.

Gray A (1967). Pseudo-Riemannian almost product Manifolds and submersions, Mich. Math. J. 16, pp. 715-737.

Mizusawa H and Koto S (1960). Holomorphically projective curvature tensors in certain almost Kaehlerian spaces, J. Fac. Sci. Niigata Univ. 2. pp. $33-43$.

Negi U S and Semwal Ashish (2011). The study on almost Kaehlerian conformal recurrent and symmetric Manifolds, International journal of mathematical Archive- 2(9), pp. 1666-1671.

Prvanovic M and Pusic N (1995). On Manifolds admitting some semi-symmetric metric connection, Indian Journal of Math. 37(1), pp. 37-67.

Yano K (1965). Differential Geometry on Complex and Almost Complex spaces. Pergamon Press, London, 